\section{Perceived stigma and depression among caregivers of patients with bipolar disorder}

\author{
DEBOR AH A. PERLICK, DAVID J. MIKLOWITZ, BRUCE G. LINK, \\ ELMER STRUENING, RICHARD KACZYNSKI, JODI GONZALEZ, \\ LAUREN N. MANNING, NANCY WOLFF and ROBERT A. ROSENHECK
}

\begin{abstract}
Summary This study investigates the associations between perceived stigma, depressive symptoms and coping among caregivers of people with bipolar disorder. Caregivers of 500 people with DSM-IV bipolar disorder responded to measures of these constructs at study entry. Patients' clinical and functional status were evaluated within 30 days of the caregiver assessment. Perceived stigma was

positively associated with caregiver depressive symptoms, controlling for patient status and socio-demographic factors. Social support and avoidance coping accounted for $63 \%$ of the relationship between caregiver stigma and depression. Results suggest that caregivers' perceptions of stigma may negatively affect their mental health by reducing their coping effectiveness.
\end{abstract}

Declaration of interest None. This study was supported by grants $\mathrm{MH}-65015$ and NIMH-800I from the National Institute of Mental Health.

Studies have shown that $43 \%$ to $92 \%$ of caregivers of people with mental illness report feeling stigmatised (e.g. Struening et al, 2001) and that perceived stigma is associated with reports of depressive symptoms (e.g. Phelan et al, 1998). This study evaluates the roles of caregiver social support and avoidance coping in mediating a hypothesised association between perceived stigma and depressive symptoms among caregivers of patients with bipolar disorder. Because perceived stigma has been associated with withdrawal from supports (e.g. Fadden et al, 1987), and social support buffers against recurrence of depression (e.g. Brown \& Harris, 1978), we hypothesised that social support might mediate the association between perceived stigma and depression. Similarly, because avoidance coping has been associated with depression (Powers et al, 2002), we hypothesised that it might mediate the association between stigma and depression. We predicted that perceived stigma and avoidance coping would be positively associated - and social support inversely associated - with depressive symptoms after controlling for socio-demographic factors and patient clinical status.

\section{METHOD}

Participants were the primary caregivers of 500 patients with bipolar disorder enrolled in the Systematic Treatment Enhancement Program for Bipolar Disorder (Sachs et al, 2003). All patients recruited from 1 August 2002 through 31 December 2003 at each of eight sites $(n=778)$ were invited to participate; $676(87 \%)$ agreed to do so and identified their primary caregiver using the method established by Pollak \& Perlick (1991). Almost three-quarters (74\%; $n=500)$ of the 676 caregivers invited to participate agreed. Caregivers who participated did not differ statistically from those who declined on age, gender, education, marital status, relationship to the patient and co-residence.

Patient lifetime diagnosis was based on a standardised Affective Disorder Evaluation (Sachs et al, 2003), comprising a modified version of the mood and psychosis modules from the Structured Clinical Interview for DSM-IV diagnosis; First et $a l, 1996)$ and the Mini International Neuropsychiatric Interview (Sheehan et al, 1998). The Clinical Monitoring Form, a semi-structured interview, was administered by the treating psychiatrist to evaluate the presence or absence of a DSM-IV episode of affective illness within the past 30 days (Sachs et al, 2003). The Global Assessment of Functioning (GAF) score
(American Psychiatric Association, 1994) represented patients' functional status over this time frame. Caregivers were interviewed within 30 days (mean 6 days, s.d. $=25.5$, median 3.0) of a patient assessment by trained research assistants.

Caregiver perceptions of stigma were assessed using the Devaluation of Consumer Families Scale (Struening et al, 2001), a seven-item Likert scale (rating of statements such as 'Most people look down on families that have a member who is mentally ill'), summed to yield a total stigma score (Cronbach's $\alpha=0.80$ ), with higher scores indicating greater stigma. Caregiver depressive symptoms were assessed using the Center for Epidemiological Studies Depression scale (CES-D; Radloff, 1977) (Cronbach's $\alpha=0.90$ ). Avoidance coping was assessed using the sub-scale from Scazufca \& Kuipers's (1999) measure. Items describe a cognitive or behavioural strategy for coping with a stressful event, e.g. 'Avoided other people'. Caregivers rated how often they used each strategy to handle the most stressful situation with their relative in the past month on fivepoint Likert scales, with higher scores indicating higher avoidance. The 11-item Abbreviated Duke Social Support Index (ADSSI; Koenig et al, 1993) was used to measure subjective support and social interaction; higher numbers represented higher levels of support.

\section{RESULTS}

Using a hierarchical regression model, the CES-D was regressed on sociodemographic variables (step 1), caregiver stigma (step 2), patient clinical status (step 3) and hypothetical mediating variables (steps 4,5 ) (see data supplement to online version of this paper). Caregiver stigma was significantly and positively associated with CES-D score and contributed an additional $4 \%$ variance after controlling for socio-demographic factors ( $F$ change $_{1,337}=14.09, P<0.001$ ). Patient status was not significantly associated with CES-D score. The unique contribution of stigma was reduced by over $50 \%$ with the entry of social support measures (step 4), indicating these explained about half of the contribution of stigma to CES-D score. The addition of social support and avoidance coping measures (step 5) increased the variance explained by $18 \%$ and $6 \%$ respectively. The combined effect of 
social support and avoidance coping explained nearly two-thirds $(63 \%)$ of the contribution of stigma to CES-D score. The significant associations of the social support measures and avoidance coping with CES-D score and resulting decrease in the associations between stigma and CES-D, coupled with significant associations between stigma and social support (subjective support, Pearson $r=-0.24$; instrumental support, Pearson $r=-0.19$ ) and between stigma and avoidance coping (Pearson $r=0.18$; all $P$ values $<0.001$ ) support the hypothesised mediating effects of social support and avoidance coping in the relationship between perceived stigma and depressive symptoms (Barron \& Kenney, 1986). Exploratory analyses found that the overall pattern of associations between caregiver stigma, support and coping, and CES-D scores did not differ for caregivers in different age groups, of different gender and with different relationships to the patient.

\section{DISCUSSION}

Our finding that greater perceptions of stigma towards caregivers were associated with significantly higher levels of depressive symptoms suggests that in addition to posing a barrier to the recovery of people with mental illness, stigma erodes the morale of the family members who help care for them. The finding that social support and avoidance coping together largely explained the association between stigma and depression offers a plausible explanation for the depressive symptoms commonly reported by up to $40 \%$ of caregivers of people with mental illness (Dyck et al, 1999): caregivers may retreat from social support and adopt avoidance coping in order to fend off anticipated rejection and/or embarrassment. Because social support is a well-established buffer against recurrence of depression, withdrawal from potential supporters as an adaptation to stigmatisation illustrates the double jeopardy confronting caregivers of patients with mental illness.

Because this study uses cross-sectional data we cannot make causal inferences. Although it is possible that stigmatisation might engender feelings of hopelessness, it is also possible that caregiver depression in bipolar disorder is at least in part due to genetic transmission of this illness. However, recurrence of depression has been linked to psychosocial stressors such as

DEBORAH A. PERLICK, PhD, Department of Psychiatry, Yale University School of Medicine, New Haven Connecticut; DAVID J. MIKLOWITZ, PhD, Department of Psychology, University of Colorado at Boulder, Colorado; BRUCE G. LINK, PhD, ELMER STRUENING, PhD, Mailman School of Public Health of Columbia University, New York; RICHARD KACZYNSKI, PhD, Department of Psychiatry, Yale University School of Medicine, New Haven, Connecticut; JODI GONZALEZ, PhD, Department of Psychiatry, University of Texas, San Antonio, Texas; LAUREN N. MANNING, BA, Brigham \& Women's Hospital, Harvard Medical School, Boston, Massachusetts; NANCY WOLFF, PhD, School of Planning and Public Policy, Rutgers University, New Brunswick, New Jersey; ROBERT A. ROSENHECK, MD, Department of Psychiatry, Yale University School of Medicine, New Haven, Connecticut, USA

Correspondence: Dr D. A. Perlick, Mount Sinai School of Medicine, One Gustave L. Levy Place, New York, NY 10029, USA. Tel: + I 7185849000 , ext. 5231; fax: + 1718364 3576; email: deborah.perlick@mssm.edu

(First received I5 December 2005, final revision 16 January 2007, accepted I February 2007)

negative life events (Johnson \& Roberts, 1995) and caregiving strain (Russo et al, 1995), and contending with social rejection and attendant feelings of shame and embarrassment constitutes a major caregiving strain (Perlick et al, 2004). Longitudinal studies including caregivers with and without a history of affective disorder, and using clinically validated measures of depression, are needed to disentangle the relative contributions of pre-existing affective illness and societally based discrimination to caregiver depression. The potential for recurrence of depressive symptoms among the family members of patients with bipolar disorder in relation to the stresses of mental illness stigma underscores the seriousness of this social problem and the need for investigations of effective interventions to enhance caregiver coping.

\section{REFERENCES}

Americna Psychiatric Association (1994) Diagnostic and Statistical Manual of Mental Disorders (4th edn) (DSM-IV). Washington, DC: APA

Brown, G.W. \& Harris, T. O. (1978) Social Origins of Depression: A Study of Psychiatric Disorder in Women. Free Press.

Barron, R. M. \& Kenny, D. A. (1986) The moderatormediator variable distinction in social psychological research: conceptual, strategic, and statistical considerations. Journal of Personality and Social Psychology, 5I, II73-1182.

Dyck, D. G., Short, R. \& Vitaliano, P. P. (1999) Predictors of burden and infectious illness in schizophrenia caregivers. Psychosomatic Medicine, $6 \mathbf{1}$ $4 \mid 1-419$

Fadden, G., Bebbington, P. \& Kuipers, L. (1987) The burden of care: the impact of functional psychiatric illness on the patient's family. British Journal of Psychiatry, I50, 285-292

First, M. B., Spitzer, R. L., Gibbon, M. B., et al (1996) Structured Clinical Interview for Axes I and II DSM-IV Disorders - Patient Edition (SCID-IP). Biometrics Research Department, New York State Psychiatric Institute.
Hollingshead, A. \& Redlich, F. C. (1958) Social Class and Mental Illness: A Community Study. Wiley.

Johnson, K. R. \& Roberts, J. E. (1995) Life events and bipolar disorder: implications from biological theories. Psychological Bulletin, II7, 434-449.

Koenig, H. G., Westlund, R. E., George, L. K., et a (1993) Abbreviating the Duke Social Support Index fo use in chronically ill elderly individuals. Psychosomatics, 34, 6I-69.

Perlick, D. A., Rosenheck, R. A., Clarkin, J. F., et al (2004) Impact of family burden and affective response on clinical outcome among patients with bipolar disorder. Psychiatric Services, 55, 1029-1035.

Phelan, J. C., Bromet, E. J. \& Link, B. G. (1998) Psychiatric illness and family stigma. Schizophrenia Bulletin, 24, 115-126.

Pollak, C. P. \& Perlick, D. (199I) Sleep problems and institutionalization of the elderly. Journal of Geriatric Psychiatry and Neurology, 4, 204-210.

Powers, D. V., Gallagher-Thompson, D. \& Kraemer H. C. (2002) Coping and depression in Alzheimer's caregivers: longitudinal evidence of stability. Journal of Gerontology Series B: Psychological Science and Social Science, 57, 205-2II.

Radloff, L. S. (1977) The CES-D scale: a self-report depression scale for research in the general population Applied Psychological Measure, I, 385-40I.

Russo, J., Vitaliano, P. P., Brewer, D. D., et al (1995)

Psychiatric disorders in spouse caregivers of care recipients with Alzheimer's disease and matched controls: a diathesis-stress model of psychopathology Journal of Abnormal Psychology, 104, 197-204.

Sachs, G. S., Thase, M. E., Otto, M. W., et al (2003) Rationale, design, and methods of the systematic treatment enhancement program for bipolar disorder (STEP-BD). Biological Psychiatry, 53, 1028-1042.

Scazufca, M. \& Kuipers, E. (1999) Coping strategies in relatives of people with schizophrenia before and after psychiatric admission. British Journal of Psychiatry, 174, 154-158.

Sheehan, D. V., Lecrubier, Y., Sheehan, K. H., et al (1998) The Mini-International Neuropsychiatric Interview (M.I.N.I.): the development and validation of a structured diagnostic psychiatric interview for DSM-IV and ICD-10. Journal of Clinical Psychiatry, 59 (Suppl. 20), 22-33.

Struening, E. L., Perlick, D. A., Link, B. G., et (200I) The extent to which caregivers believe most people devalue consumers and their families. Psychiatric Services, 52, 1633-1638. 